\title{
Self representation in children with emotional and behavioural disorders
}

\author{
Priya Kayastha Anand ${ }^{1}$, Uma Hirisave ${ }^{2}$ \\ ${ }^{1}$ Clinical Psychologist, Bengalaru. \\ ${ }^{2}$ Professor, Department of Clinical Psychology, National Institute of Mental Health and Neurosciences (NIMHANS) \\ - INI, Bangalore. \\ E-mail-priyakayastha@gmail.com
}

\begin{abstract}
Background: The attachment theorists have provided insights into how the representation model of attachment forms the foundation for child's early learning about self. Western studies have explored the relation between attachment and self in children. However, there is hardly any Indian research on attachment and self representation in young children.

Objective: The present study examined the self representation in children with emotional and behavioral disorders and group of normal children.

Method: Sample of 60 children aged 6-12 years were assessed on two measures namely Incomplete Doll Stories \& Self Interview. The study adopted a cross-sectional design.

Results: The findings indicate that on interview children with emotional and behavioral disorders report a secure attachment. However, on semi-projective technique they represent themselves in negative manner in context of the relationship with mother compared to the normal children. They were also found to have poor problem solving skills.

Conclusions: Methods like the incomplete doll stories may help one understand facets of the self representation of children that may not be revealed on direct interview.
\end{abstract}

Key words: self representation, attachment, emotional and behavioral disorders, doll stories

(Paper received $-27^{\text {th }}$ March 2016, Peer review completed $-10^{\text {th }}$ May 2016, Accepted $-25^{\text {th }}$ May 2016)

\section{INTRODUCTION}

Attachment theory has led to a deeper understanding of the early caregiving relationship and how it supports the early child development. In young children, attachment figure serves as a potential source to learn about self. Bowlby proposed that the representational model of the attachment figure is closely intertwined with the representational model of self [1]. Elaborating on Bowlby's ideas, Sroufe and Fleeson [2] proposed that child's early learning about self occurs in the context of his/ her relationship with attachment figure. The components of attachment figure get incorporated into the self through the process of child's learning within the relationship. Thus, the quality or security of the representation of attachment relationship is entangled with the quality of the overall representation of self in children [3]. According to Harter [4], the self feelings are reflections of attachment figure appraisal of self. Thus, children who experience parents as responsive, loving and supportive develop a model of self characterized as loveable and competent and those who experience parents as rejecting and unsupportive develop model of self as unlovable, incompetent and generally unworthy. 
The Indian research has explored attachment in relation to other variables such as temperament [5], socioemotional behavior [6] and psychopathology [7]. Janveja [8] has reported negative internal representation in 4-8 years old children diagnosed with behavioral disorder. There are no Indian studies on self representation in normal children as well as in children with emotional and behavioral disorders.

The present study was carried out to compare self representation in children with emotional and behavioral disorders with group of normal children using interview method and a semi projective test. The research protocol was approved by the departmental protocol review committee and informed consent was given by the parents of the children.

\section{METHODOLOGY}

\section{Sample and study design}

The study sample consisted of sixty children aged 6-12 years. The clinical group comprised of 30 children with ICD-10 [9] diagnosis of Behavioral \& Emotional Disorder (F.90-F.98), Depressive Disorder (F.32, F.33), Dissociative Disorder (F.44) and with / without co-morbid diagnosis of Specific Developmental Disorder of scholastic skills (F.81). The data was collected from children and mothers attending Child \& Adolescent Psychiatry Services of National Institute of Mental Health \& Neuro Sciences (NIMHANS), Bengaluru, India.

The normal group consisted of 30 children selected from school catering to middle socio economic status in the city of Bengaluru. These children were frequency matched with clinical group with respect to age and gender. The children in both the groups came from intact families and did not have any chronic physical or neurological disorder as reported by teachers / parents. The design adopted in the study was cross-sectional in nature.

\section{Measures}

The assessment included semi-structured interview with the parent to obtain demographic and background details; Developmental Psychopathology Checklist (DPCL) [10] and Child Behavior Questionnaire (CBQ) [11] for screening emotional and behavioral problems; and the child views of the self on Incomplete Doll Stories (IDS) [3] and Self Interview (SI) [3]. DPCL was administered to mothers of children in clinical group to obtain information on psychological problems and psychosocial stressors and CBQ was administered with teachers to obtain information on group of normal children. The IDS is a semi projective test consisting of six stories in which child is provided with family of dolls and asked to narrate a story. The stories tap the child views of the self as participating within the relationship with the attachment figure. For the purpose of assessment, only three stories pertaining to relationship acknowledging interaction between mother and child were included. Each of the stories was rated on five point scale, with higher score indicating secure attachment. The SI consists of 20 questions designed to reflect the child's own representation of himself/herself.

\section{STATISTICAL ANALYSIS}

Simple descriptive statistics and percentages were used in the analysis.

\section{RESULTS AND DISCUSSION}

The present study compared the self representation in children with emotional and behavioral disorders and group of normal children. The self representation was assessed on two measures namely Incomplete Doll Stories and Self Interview. Majority of the children (63\%) in both the groups were in their middle childhood. Children in the clinical group had a diagnosis of ADHD (40\%). Other diagnostic categories were dissociative disorder $(27 \%)$, emotional disorder (20\%) and conduct disorder (16\%).

On self interview, when children were asked question directly about their 'self', both group of children have represented positively. However, when a semi-projective test was used, there was a discrepancy in responses indicating better self representation in group of normal children than the clinical group. 
On self interview, the findings reveal no significant difference between the children in clinical and normal group. This indicates that majority of children in both groups represented themselves as open flexible and described themselves positively. Children in both groups described similar qualities such as scoring good marks, being obedient, not indulging in fights and helping mother as positive aspects about themselves that are liked by mother. In another study, children from lesser privileged background with emotional and behavioral problems were found to have open flexible and avoidant perfect self representation [7]. This could be attributed to factors related to socio-economic disadvantage such as poverty, punitive parenting and less emotional support from parents.

On the semi-projective test namely Incomplete Doll Stories, the first two stories dealt with emotionally charged and relationship acknowledging interaction between mother and child. In one, the child makes a gift at school and presents it to his/her mother at home and in the other story, the child approaches mother and apologizes for doing something wrong. In both these stories, the mothers of normal children were more expressive of positive emotions towards their children than the mothers of clinical group of children. The group of normal children viewed mothers more positively than children in clinical group.

In the doll story involving gift theme, the children in both groups narrated mother's acknowledgement of the gift, however, the normal children viewed mother as expressing more positive emotions towards their children than children in clinical group. In the story theme concerned with apologizing, the children in the normal group have perceived mothers to be positive whereas the children in the clinical group reported instances of mothers' behavior in a hostile and violent manner.

The insecure feelings expressed by children in the clinical group may be the result of discordant interfamilial relationship characteristic of these families. Some mothers were found to be inadequate to perform their roles and had poor communication skills in dealing with child i.e. would speak to child in sarcastic manner which would be a humiliating experience to the child. Factors such as father's absence at home, not taking responsibility might lead to role strain in mother and further affecting mother -child relationship.

The last theme of incomplete doll stories involved threat from outside the family where the child discovers his/her bicycle has been stolen by another child. In this story, the children in the normal group showed better problem solving and decision making ability than children in clinical group.

Responses by group of normal children on these three stories indicate positive perception of mother in the context of self and mother, as well as better problem solving skills. This finding is similar to the findings of Janveja [8] where more affiliative expressions were reported in play narratives of group of normal children than clinical group. Cassidy [3] has reported that normal children possess open and flexible self representation as well as adaptive competence in situations where attachment figure is not present. The findings of the present study also highlight similar adaptive competencies among group of normal children.

It is important to note the discrepancy in the findings of self representation when assessed by semiprojective test and self interview method. The children in the clinical group showed less confidence in availability and responsiveness of the mother on incomplete doll stories which was designed to reveal implicitly operating assumptions about mental representation. However, on self interview these children presented positive image of their mother who were equally loving and accepting as mother of normal group. Hence, taken as a whole, the findings suggest that the children in clinical group may be operating from two inconsistent working models of attachment figure and self. One working model when accessed through direct discussion presents attachment figure as good and supportive, whereas the other model when looked into unconscious operations through semi projective method presented mother as unresponsive and unsupportive. This lends support to Bowlby's idea that children who are not securely attached are likely to develop inconsistent working models of attachment figures and the self.

Overall, the findings of the present study indicate that the clinical group children on interview reported open flexible self representation. On the semi- projective stories completed by them reveal negative 
interaction and poor problem solving in interaction context. It is important that while assessing self representation in children both interview as well as semi-projective methods must be administered.

The small number of participants and unequal representation of gender limits the generalizibility of results. Research should focus on assessing attachment and self representation in large sample of children using both projective and interview methods. Further, the sample may include children from varied socio economic strata, institutionalized children; children reared by single parent and adopted children. Additionally, it would be interesting to investigate how peer relations, personality variables such as temperament relate to self representation.

\title{
REFERENCES
}

1. Bowlby J. Attachment and loss: Separation: Anxiety and anger (Vol. 2). New York : Basic Books ; 1973.

2. Sroufe LA, Fleeson J. An Organizational Perspective on the self. In L.A Sroufe (Eds.), Emotional Development: Organization of the emotional life in early year, New York: Cambridge University Press ; 1986.

3. Cassidy J. Child-mother attachment and the self in six-year-olds. Child development. 1988;59:121-34.

4. Harter S. The Development of Self Representations. In Damon W, Eisenberg N (Eds). Handbook of Child Psychology,Vol.3, Social, Emotional \& Personality Development,5th Edition (pp.553-617). New York: John Wiley \& Sons ; 1998.

5. Nithya Poornima M, Chitra S, Hirisave U. Attachment, temperament and social behavior in early childhood. Indian J Clin Psychol 2005;32:91-7.

6. Gitanjali M. Attachment patterns \& socio- emotional behavior in middle childhood. Unpublished M.Phil Dissertation, NIMHANS, Bengaluru ; 2004.

7. Vishwanatha K, Hirisave U. Attachment and psychopathology in children with emotional disorders. Indian J Pediatrics 2008;75(4):395-7.

8. Janveja V. Teddy Bear's Picnic: Personal constructs as revealed in play of children with behavioral problems. Unpublished M.Phil Dissertation, NIMHANS, Bengaluru ; 1999.

9. World Health Organization. International Classification of Diseases $-10^{\text {th }}$ edition ; 1992.

10. Kapur M, Barnabas I, Reddy MV, Rozario J, Uma H. Development of a checklist for assessment of childhood psychopathology in the Indian setting. Indian J Clin Psychol 1994;21:40-52.

11. Rutter M. A children's behavior questionnaire for completion by teachers: Preliminary findings. J Child Psychol Psychiatry 1967;8:1-12.

\author{
Acknowledgements - Nil \\ Source of Funding - Nil \\ Conflict of Interest - Nil
}

